\title{
Flames in Type Ia Supernova: Deflagration-Detonation Transition in the Oxygen Burning Flame
}

\author{
S. E. Woosley ${ }^{1}$, A. R. Kerstein ${ }^{2}$, and A. J. Aspden ${ }^{3}$
}

\begin{abstract}
The flame in a Type Ia supernova is a conglomerate structure which, depending on density, may involve separate regions of carbon, oxygen and silicon burning, all propagating in a self-similar, subsonic front. The separation between these three burning regions increases as the density declines until eventually, below about $2 \times 10^{7} \mathrm{~g} \mathrm{~cm}^{-3}$, only carbon burning remains active, the other two burning phases having "frozen out" on stellar scales. Between 2 and $3 \times 10^{7} \mathrm{~g}$ $\mathrm{cm}^{-3}$ however, there remains an energetic oxygen burning region that trails the carbon burning by an amount that is sensitive to the turbulence intensity. As the carbon flame makes a transition to the distributed regime (Karlovitz number $\gtrsim 10$ ), the characteristic separation between the carbon and oxygen burning regions increases dramatically, from a fraction of a meter to many kilometers. The oxygen-rich mixture between the two flames is created at a nearly constant temperature, and turbulence helps to maintain islands of well-mixed isothermal fuel as the temperature increases. The delayed burning of these regions can be supersonic and could initiate a detonation.
\end{abstract}

Subject headings: supernovae: general; hydrodynamics, shock waves, turbulence

\section{INTRODUCTION}

Currently favored models for Type Ia supernovae that involve the explosion of a carbonoxygen white dwarf as it approaches the Chandrasekhar mass require a late time transition of the thermonuclear burning to a detonation wave (e.g., Hoflich et al. 1995; Mazzali et al.

\footnotetext{
${ }^{1}$ Department of Astronomy and Astrophysics, University of California, Santa Cruz, CA 95064; woosley@ucolick.org

${ }^{2}$ Combustion Research Facility, Sandia National Laboratories, Livermore, CA 94551; arkerst@sandia.gov

${ }^{3}$ Center for Computational Sciences and Engineering, Lawrence Berkeley National Laboratory, CA; ajaspden@lbl.gov
} 
2007; Kasen et al. 2009). That transition needs to occur after the white dwarf has expanded appreciably, so that sufficient material burns to intermediate mass elements, especially silicon and calcium, and not all the way to iron.

The mechanism for this transition is both complex and controversial. It could involve a mechanical collision and compression, as in the "gravitationally confined detonation" model (Plewa et al. 2004), or it might happen spontaneously as turbulence mixes hot ashes with cold fuel at low density (Khokhlov, Oran, \& Wheeler 1997; Niemeyer \& Woosley 1997). We have previously studied the possibility of a delayed transition to detonation in the carbon flame (Woosley et al. 2009, Paper 1). Here we explore the possibility of detonation in the oxygen flame that follows behind. We find that detonation is possible for a narrow range of densities that depends upon the composition and turbulent intensity. For typical values, the transition density $\rho \approx 2.5 \times 10^{7} \mathrm{~g} \mathrm{~cm}^{-3}$. Detonation at such a high density would tend to give a bright supernova.

\section{TURBULENT FLAMES IN TYPE Ia SUPERNOVAE}

\subsection{The Carbon Flame}

Nuclear burning in a Type Ia supernova that does not promptly detonate involves some very interesting combustion physics. Turbulence, generated by rising plumes of ash, interacts with a flame whose width and speed vary as the density declines. Moreover, the flame is not a single stage affair, but a stacked structure in which, depending on the density, up to three discrete stages of burning occur - carbon, oxygen and silicon burning. In Paper 1, we considered densities sufficiently low that silicon burning had gone out and the oxygen flame lay far behind the carbon flame (farther than the integral scale of the turbulence). This allowed us to treat, in some detail, the interaction of single stage carbon-burning flames with background turbulence having specified properties.

In the simplest case, sometimes referred to as the "flamelet regime", laminar flames are folded by the turbulence to form a "flame brush", but preserve their individual identity. That is, viewed on a small scale, each flame still propagates with a well-defined speed and width given by conduction (e.g. Landau \& Lifshitz 1959). In terms of combustion characteristics, the Gibson scale is larger than the flame thickness and the Karlovitz number, Ka, is less than 1. Sufficient folding occurs that the overall flame brush moves along with a speed sufficient to digest the material entrained by the eddies on the integral scale. That is, the overall speed of the flame is approximately $\mathrm{U}_{t}$, with $\mathrm{L}$, the integral scale of the assumed (homogeneous, isotropic, Kolmogorov) turbulence and $\mathrm{U}_{t}$, its characteristic speed on that scale (Damköhler 
1940). This sort of burning characterizes most of the sub-sonic phase of the supernova when the density is above a few times $10^{7} \mathrm{~g} \mathrm{~cm}^{-3}$.

At lower densities however, the carbon flame becomes thicker and slower, and eventually is torn by turbulence. For conditions of interest in this paper, $2 \lesssim \rho / 10^{7} \mathrm{~g} \mathrm{~cm}^{-3} \lesssim 3, \mathrm{~L} \sim 10$ $\mathrm{km}, \mathrm{U}_{t} \sim 500 \mathrm{~km} \mathrm{~s}^{-1}$, and a carbon mass fraction of $40 \%$, carbon burns in the distributed regime with a $\mathrm{Ka} \gg 1$, (Aspden et al. 2008). This can be inferred from Table 3 of Paper 1 and the scaling relation $\mathrm{Ka} \propto U_{t}^{3 / 2}$. For $\mathrm{U}_{t} \approx 500 \mathrm{~km} \mathrm{~s}^{-1}, \mathrm{Ka} \sim 10$ for the conditions of interest. In this regime, the carbon flame moves with a speed near $\mathrm{U}_{t}$ and its turbulently broadened width is of order L, though there will occasionally be factors of several variation in both quantities and carbon may burn simultaneously at several locations within the integral scale.

\subsection{The Oxygen Flame}

The carbon flame will be followed, at some distance, depending upon density and turbulence parameters, by a region where oxygen burns to silicon and heavier elements. Silicon burning can be neglected. The oxygen flame is different from the carbon flame in that it is "slaved" to the carbon flame, and its speed is never determined by conduction in the oxygen ashes. For small values of turbulent energy, oxygen burns strictly inductively. That is, carbon burning, for a given initial temperature and density, produces a unique oxygen-rich composition at a unique temperature and density. For these conditions, oxygen has a well determined time scale to burn isobarically. That time scale multiplied by the speed of the carbon flame gives the width of the oxygen burning region.

Values of the oxygen burning time scale scale, $\tau_{16}$, can be evaluated numerically using a 7 isotope network similar to that of Timmes et al. (2000) (see also Paper 1). A representative sample is given as a function of carbon fuel density and mass fraction in Table 1. A mixture of carbon and oxygen with a given initial density and a temperature of $6 \times 10^{8} \mathrm{~K}$ is allowed to evolve isobarically until the carbon, oxygen and neon abundances are all quite small. The equation of state and heat capacity are taken from Timmes \& Swesty (2000) and electron screening corrections are included in the reaction rates. $\tau_{16}$ is defined as the time that it takes the mass fraction of ${ }^{16} \mathrm{O}$ to decline from its maximum value following carbon and neon burning $\left(\mathrm{X}_{16}\right.$ in the table) to a final mass fraction less than $1 \%$. This time scale is shorter than the instantaneous burning time scale, $X_{16} /\left(d X_{16} / d t\right)$, evaluated at the time of carbon

and neon depletion. That is because the temperature continues to rise as the oxygen burns and the extreme temperature sensitivity of the reaction rate leads to an acceleration of the burning. 
Oxygen burns away at a distance behind the carbon flame given by

$$
\delta_{16}=S_{12} \tau_{16},
$$

where $\tau_{16}$ is determined by the (carbon-burning) fuel density and temperature. In fact, due to the temperature sensitivity of the oxygen burning rate, most of the oxygen burns at about this distance behind the carbon flame. This quantity, $\delta_{16}$, can thus be thought of as the width of the oxygen flame. Note that $S_{12}$, the velocity of the carbon flame, could describe a single laminar flame or a collection of carbon flamelets so long as the distribution had a total width less than $\delta_{16}$.

In the oxygen, as in the carbon, there is a also characteristic length scale,

$$
\lambda_{16}=\left(\epsilon \tau_{16}^{3}\right)^{1 / 2}=L / \mathrm{Da}_{16}{ }^{3 / 2},
$$

which is the typical size of a region that can mix by turbulent diffusion on a nuclear burning time (Paper 1). Here $\epsilon$ is the energy density in the turbulent cascade, $\epsilon=U^{3}(L) / L$, and $\mathrm{Da}_{16}$ is a Damkohler-like variable for the oxygen,

$$
\mathrm{Da}_{16}=\frac{\mathrm{L}}{U_{\mathrm{L}} \tau_{16}} .
$$

The behavior of the oxygen flame is sensitive to the relative sizes of the three length scales, $\delta_{16}, \lambda_{16}$, and L.

The most interesting cases for SN Ia involve either laminar burning (the trivial case $\left.\lambda_{16}=0 ; \S 3.1\right)$ or $S_{12}=U_{t}$. As we shall see in $\S 3$, the latter case is realized to within a factor of a few in cases where turbulence dominates the burning (see also Paper 1). The flame brush advances into a fuel with a speed given approximtely by the characteristic speed of the turbulence on the integral scale. This results in a great simplification of the problem since, if $S_{12}=U_{t}$, then $\delta_{16}=U_{t} \tau_{16}$, and

$$
\begin{aligned}
& \frac{L}{\delta_{16}}=\mathrm{Da} \\
& \frac{L}{\lambda_{16}}=\mathrm{Da}^{3 / 2} \\
& \frac{\delta_{16}}{\lambda_{16}}=\mathrm{Da}^{1 / 2} .
\end{aligned}
$$

The nature of the solution is specified completely by the Damhohler number. These equations also imply that $\delta_{16}$ will always lie between $\lambda_{16}$ and $\mathrm{L}$.

For $\mathrm{Da} \ll 1$, we shall find that turbulence does not greatly alter the overall oxygen flame width, which remains close to $\delta_{16}(\S 3.2)$. Turbulence can broaden the oxygen burning 
region a little though. Since the reaction rate is very temperature sensitive, burning in an isothermal layer is generally slower than in one with a the same heat content and a temperature gradient. Since $\mathrm{Da}<1$ implies $L<\delta_{16}<\lambda_{16}$, mixing by eddies will always be faster than burning within the oxygen-rich region.

For $\mathrm{Da} \gg 1$, there can be multiple oxygen flames, in conjunction with multiple carbon flames. This region is not of interest here since each of the flamelets would be thinner in the multiple flame case and we are seeking the largest possible single, homogeneous region that might trigger a runaway.

The most interesting case occurs when Da $\gtrsim 1$ ( $\S 3.3)$. Then $\mathrm{L}, \lambda_{16}$, and the oxygen flame width are all comparable in size. Burning goes on in a single or very few oxygen flames and each has the potential to be well mixed. The condition $\mathrm{Da}_{16}=1$, or $U_{t} \tau_{16}=L$ gives, for an assumed $\mathrm{U}_{t}$ and initial composition, a unique density. For turbulence with $\mathrm{U}_{t}=500 \mathrm{~km} \mathrm{~s}^{-1}$ on a length scale of $10 \mathrm{~km}$, the oxygen burning time should be $20 \mathrm{~ms}$ which for a carbon mass fraction of $40 \%$ implies a density (Table 1) near $2.5 \times 10^{7} \mathrm{~g} \mathrm{~cm}^{-3}$. For less intense turbulence, supersonic burning will be difficult to achieve. Much larger values (by a factor of two) are difficult to achieve in the star (Röpke 2007). So the region of interest for oxygen detonation is well determined. Interestingly, lower carbon mass fractions imply that favorable conditions for detonation are achieved at higher density. Since ignition density correlates positively with peak luminosity (e.g. Kasen et al. 2009), a brighter supernova might be favored by a more carbon-poor (i.e., oxygen-rich) composition.

\section{CALCULATIONS USING THE LINEAR EDDY MODEL (LEM)}

To illustrate the possibilities and explore the potential for detonation in the oxygen flame, we carried out simulations of turbulent burning similar to those done for carbon burning in Paper 1. We again used the Linear Eddy Model (LEM Kerstein 1991) to represent the interaction of turbulence and burning on a one-dimensional grid. The LEM code includes diffusive transport, here represented by a turbulent diffusion coefficient, nuclear burning calculated using a 7 isotope network, the effects of turbulent eddy motions, and an equation of state (Timmes \& Swesty 2000). The nuclear reaction network which includes helium, ${ }^{12} \mathrm{C}$, ${ }^{16} \mathrm{O},{ }^{20} \mathrm{Ne},{ }^{24} \mathrm{Mg},{ }^{28} \mathrm{Si}$, and ${ }^{56} \mathrm{Ni}$, is adequate to represent realistically the effects of carbon, neon and oxygen burning. Electron screening corrections are included in the reaction rates.

We calculated both the carbon and oxygen burning flame on the same grid using the LEM code and techniques described in Paper 1. As in Paper 1, the turbulent eddy frequency factor, C, was set to 5 . 

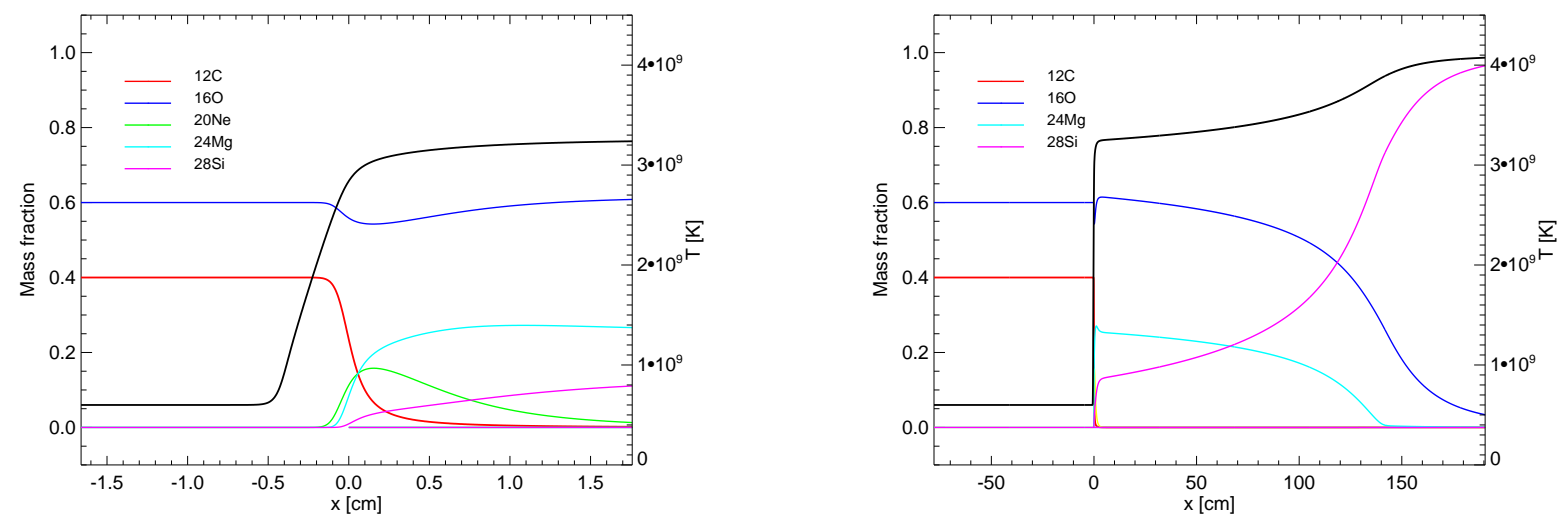

Fig. 1. - Two stage carbon and oxygen burning flame in the laminar regime. The initial density is $2.5 \times 10^{7} \mathrm{~g} \mathrm{~cm}^{-3}$ and the composition is $60 \%$ oxygen and $40 \%$ carbon by mass. The laminar speed is $1.3 \times 10^{4} \mathrm{~cm} \mathrm{~s}^{-1}$ and the oxygen burning flame is slaved to the carbon burning flame. Neon briefly burns behind the carbon flame.

\subsection{Laminar two-stage flames}

For a typical density of interest in the supernova, $2.5 \times 10^{7} \mathrm{~g} \mathrm{~cm}^{-3}(\S 2.2)$, Fig. 1 shows the structure of a two-component (carbon and oxygen) laminar flame in the absence of any turbulence. Carbon and neon burning produce an oxygen-rich composition $\left(62 \%{ }^{16} \mathrm{O}, 27 \%\right.$ ${ }^{24} \mathrm{Mg}$, and $11 \%{ }^{28} \mathrm{Si}$ ) with a temperature $3.18 \times 10^{9} \mathrm{~K}$ and density $1.66 \times 10^{7} \mathrm{~g} \mathrm{~cm}^{-3}$. The laminar speed of the carbon flame is $1.3 \times 10^{4} \mathrm{~cm} \mathrm{~s}^{-1}$. Isobaric burning of oxygen behind the carbon flame (pressure $=3.233 \times 10^{24}$ dyne $\mathrm{cm}^{-2}$ ) takes $14.8 \mathrm{~ms}$ (Table 1 ). The oxygen thus burns away in a distance $(0.0148)(13000)=190 \mathrm{~cm}$ behind the carbon flame.

\subsection{Oxygen Flames in the Well-Stirred Reactor Regime - $\mathrm{Da}_{16}<1$}

The well-stirred reactor regime (Peters 1986) is characterized by a Damkohler number less than one and a large Karlovitz number (Kerstein 2001; Woosley et al. 2009). In this regime, the oxygen burns as a single turbulently broadened flame structure whose speed and width are determined by the carbon flame speed at those conditions. Fig. 2 shows an example of an oxygen flame in this regime.

The turbulence here has a dissipation rate, $\epsilon=U_{t}^{3} / L=10^{15} \mathrm{erg} \mathrm{g}^{-1} \mathrm{~s}^{-1}$, that might be typical for a SN Ia in mildly turbulent region $\left(\epsilon=10^{15} \mathrm{erg} \mathrm{g}^{-1} \mathrm{~s}^{-1}\right.$ would also be characteristic of turbulence with $\mathrm{U}_{t}=100 \mathrm{~km} \mathrm{~s}^{-1}$ on a length scale of $10 \mathrm{~km}$ ), but the integral scale has 


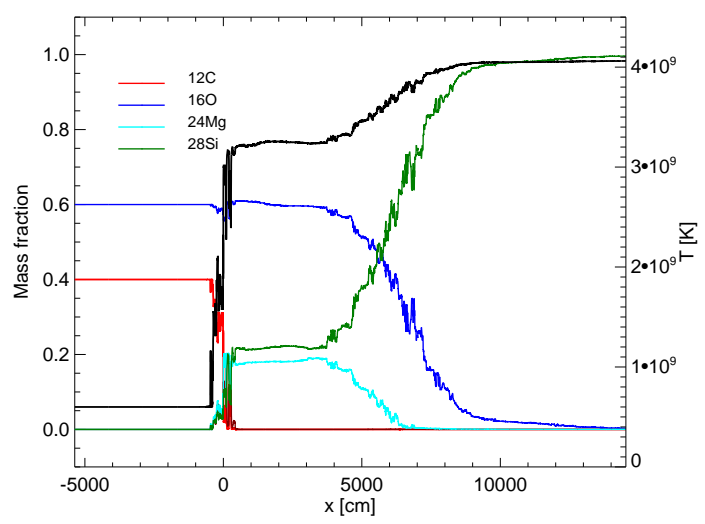

Fig. 2.- The same flame as in Fig. 1 but in the presence of turbulence with a characteristic speed of $7.94 \times 10^{5} \mathrm{~cm} \mathrm{~s}^{-1}$ on an integral scale of $500 \mathrm{~cm}$, i.e, $\epsilon=U_{t}^{3} / L=10^{15} \mathrm{erg} \mathrm{g}^{-1}$ $\mathrm{s}^{-1}$. The $300 \mathrm{~m}$ grid here had 30,000 zones so that each zone was $1 \mathrm{~cm}$. The speed of the turbulently broadened and accelerated carbon flame speed is $5.3 \times 10^{5} \mathrm{~cm} \mathrm{~s}^{-1}$. The width of the oxygen flame is this new speed times the nuclear time scale, $14.8 \mathrm{~ms}$ (Table 1) or 7800 $\mathrm{cm}$, larger than the integral scale. Hence the effective Damkohler number is less than 1 . The unburned oxygen layer is also orders of magnitude larger than in Fig. 1.

been adjusted to be smaller than the oxygen flame thickness, specifically $500 \mathrm{~cm}$. Because of the variable temperature, the eddy size that turns over in one burning time, $\lambda_{16}$, depends on the location within the oxygen flame and is not well determined, but for a burning time scale characteristic of the whole region $(14.8 \mathrm{~ms})$ is about $6 \times 10^{4} \mathrm{~cm}$. Burning the last $30 \%$ of the oxygen (roughly half) takes only 4 ms however, so $\lambda_{16}$ in that region is $8.0 \times 10^{3} \mathrm{~cm}$. This implies a Damkohler number $0.04-0.16$.

While such small integral scales do not typify supernovae, it is interesting to note here the great increase in oxygen flame thickness brought about by the increased flame speed for carbon burning. The oxygen is still depleted $14.8 \mathrm{~ms}$ behind the carbon flame, but now 14.8 ms corresponds to $7800 \mathrm{~cm}$, a factor of 40 increase over the laminar case.

\section{3. $\quad$ Oxygen Flames in the Stirred Flame Regime - $\mathrm{Da}_{16}>1$}

Finally, we consider turbulence with properties more typical of that which exists in the most turbulent regions of the supernova at $2.5 \times 10^{7} \mathrm{~g} \mathrm{~cm}^{-3}$, when detonation might actually occur. Fig. 3 shows examples of oxygen burning flames in the stirred-flame regime (Kerstein 2001) with $U_{t}=500 \mathrm{~km} \mathrm{~s}^{-1}$ on an integral scale of $10 \mathrm{~km}$. The extended ledges of nearly 
constant conditions are atypical, but are realized in at least a few percent of the simulations (see also, section 5.4.3 of Paper 1). It is here that detonation may arise ( $(4)$. Usually a more heterogeneous structure is present. The large carbon flame speed, almost $3 U_{t}$ is partly a consequence of the definition of integral scale in the LEM code and the choice of a frequency parameter $\mathrm{C}=5$ (see discussion in section 5.3.1 of Paper 1). It is also consistent with what is seen in multi-dimensional simulations (Aspden et al. 2010), but may be regarded as a relatively rare event.

For a range of nuclear time scales of $4-15 \mathrm{~ms}$, the parameter $\lambda_{16}$ in this run is $0.9-6.5$ $\mathrm{km}$ and the Damkohler number, $\mathrm{Da}_{16}=1.5$ - 5. The mixed structures in Fig. 3 are larger than $\lambda_{16}$, reflecting both earlier mixing at a lower temperature and the merger of several " $\lambda$-flames" (Aspden et al. 2010). The thickness of the oxygen burning region, about $20 \mathrm{~km}$, is still approximately $14.8 \mathrm{~ms}$ times the carbon flame speed, $1400 \mathrm{~km} \mathrm{~s}^{-1}$, though it is a bit larger in the second frame due to the homogenizing effects of the turbulent mixing ( $\S 2.2$ ) and the trabsient merger of multiple flames.

\section{Conditions for Transition to Detonation}

Table 1 gave the oxygen burning time scale and Fig. 4 shows its history in greater detail. The graph picks up the evolution of the oxygen mass fraction, burning time scale, and the temperature at the point when oxygen has already reached its maximum abundance following carbon and neon burning (Table 1). The bottom axis shows the remaining oxygen mass fraction and the solid line shows the remaining time until oxygen has burned down to 1\%. The fact that this line becomes almost level, declining by only $2 \mathrm{~ms}$ between oxygen mass fractions of 0.45 and 0.15 is of great interest. This flattening occurs because the depletion of oxygen by the ${ }^{16} \mathrm{O}+{ }^{16} \mathrm{O}$ reaction (the rate depends on $\mathrm{X}^{2}\left({ }^{16} \mathrm{O}\right)$ ) is more than compensated for by the accelerated burning caused by the increased temperature. Below an oxygen mass fraction of about 10\%, however, the situation is reversed. The temperature is no longer rising rapidly, but the oxygen mass fraction is decreasing rapidly. The time scale declines, reaching zero at 0.01 by definition.

To illustrate the use of this plot, consider a hypothetical case in which turbulence has prepared a nearly isothermal region $10 \mathrm{~km}$ across with an oxygen mass fraction of 0.40 and a temperature, $3.66 \times 10^{9} \mathrm{~K}$. The time to burn the remaining oxygen (down to mass fraction 0.01 ) is $4.7 \mathrm{~ms}$. Since the sound speed here is about $5000 \mathrm{~km} \mathrm{~s}^{-1}$, the sound crossing time is about $2 \mathrm{~ms}$ and the oxygen will apparently burn sub-sonically. However, the plot shows that the time to burn from oxygen mass fraction 0.40 to 0.10 is only $2 \mathrm{~ms}$. Half of the initial $\sim 60 \%$ of oxygen would burn supersonically. One may question the isothermal assumption. 
Just how isothermal must the plasma be? That is the point of the dashed line in Fig. 4 which shows the derivative with respect to the temperature of $\tau_{16}$. When multiplied by a range of temperatures characterizing the region, this gives the spread in runaway times. For the example just given, $d \tau_{16} / d T_{9}$ at oxygen mass fraction 0.4 is $5.2 \mathrm{~ms}\left(10^{9} \mathrm{~K}\right)^{-1}$. A spread of temperatures of $0.2 \times 10^{9} \mathrm{~K}$, i.e. 3.56 to $3.76 \times 10^{9} \mathrm{~K}$ would result in only a 1 ms variation in the runaway time.

Is it reasonable that turbulence prepare $10 \mathrm{~km}$ regions with temperature in the range roughly 3.5 - 3.7 billion K? Yes, it is. Maximum turbulent energies in the fuel are estimated, on the basis of 3D simulation by (Röpke 2007), to be around $500-1000 \mathrm{~km} \mathrm{~s}^{-1}$ on a scale of $10 \mathrm{~km}$. The turnover time on the integral scale for $500 \mathrm{~km} \mathrm{~s}^{-1}$ is thus $20 \mathrm{~ms}$; the time to burn the oxygen at at density of $2.5 \times 10^{7} \mathrm{~g} \mathrm{~cm}^{-3}$ is $14.8 \mathrm{~ms}$. Usually the gradient in oxygen abundance or the presence of complex flame structures will violate the isothermal condition. However, mixing occasionally creates ledges of nearly constant conditions (Paper 1). An example with a ledges larger than $4 \mathrm{~km}$ was shown in Fig. 3.

\section{Conclusions}

We have explored the properties of multi-stage carbon-oxygen flames in Type Ia supernovae with particular attention to the possibility that a delayed transition to detonation may occur, triggered by explosive oxygen burning in the distributed burning regime.

We find that such detonations are, in principle, possible and well worth further investigation. Future studies will need to consider the three-dimensional, compressible hydrodynamics of the mixing and explosion. In particular, when the burning speed approaches sonic values, the assumption of constant pressure made here will no longer be valid. This may actually help the detonation get started (Woosley et al. 2009). Increased pressure leads to more rapid burning and accelerates the runaway. On the other hand, the condition of supersonic burning demonstrated here is a necessary, but insufficient condition for detonation. When considered in spherical geometry, the strength of the pressure wave may diminish with increased size. The actual three-dimensional gradients of composition and especially temperature surrounding the "detonator" are also important. Finally, it has yet to be conclusively demonstrated that the necessary turbulent energies, $\epsilon=U_{t}^{3} / L \gtrsim 10^{17} \mathrm{erg} \mathrm{g}^{-1} \mathrm{~s}^{-1}$, will actually be achieved in the fuel a realistic model. The calculations of Röpke (2007) need repeating for a variety of situations, especially for the case of off-center ignition. Does isotropic, Kolmogorov turbulence with $U_{t} \gtrsim 500 \mathrm{~km} \mathrm{~s}^{-1}$ really exist on a scale of $10 \mathrm{~km}$ in the unburned fuel when a density $2.5 \times 10^{7} \mathrm{~g} \mathrm{~cm}^{-3}$ is reached? 

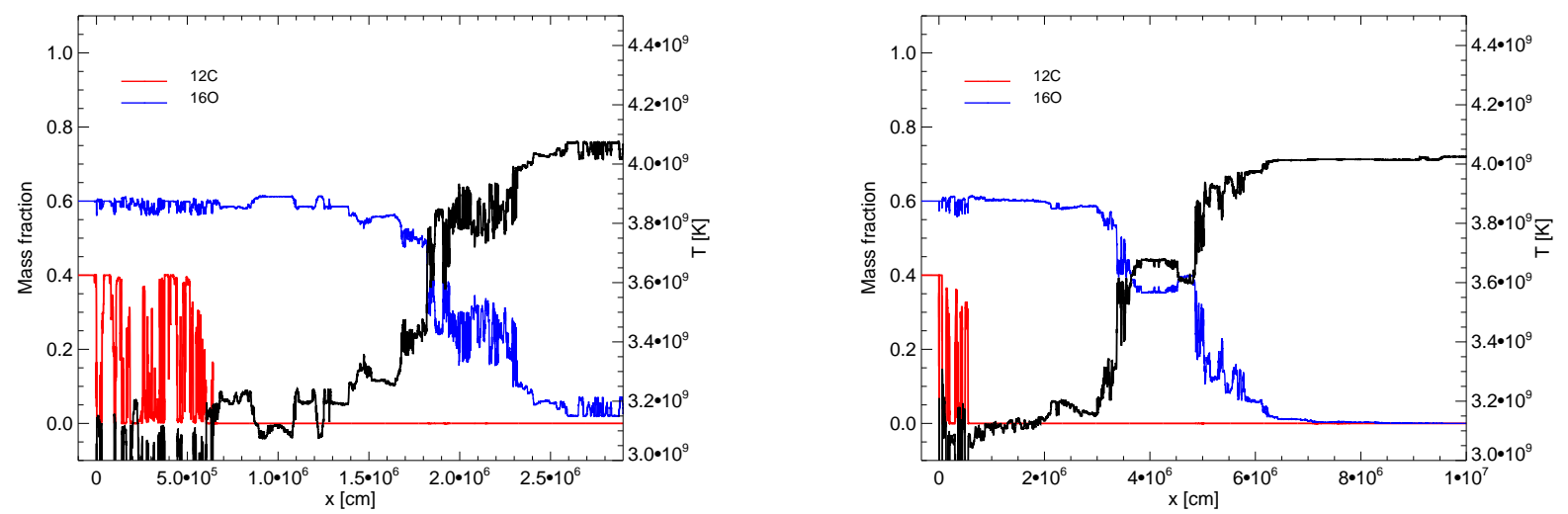

Fig. 3.- A compound carbon-oxygen flame in a turbulent medium with $U_{L}=500 \mathrm{~km} \mathrm{~s}^{-1}$ on an integral scale of $10 \mathrm{~km}\left(\epsilon=1.25 \times 10^{17}\right)$. The overall flame speed is $1400 \mathrm{~km} / \mathrm{s}$. Initial density was $2.5 \times 10^{7} \mathrm{~g} \mathrm{~cm}^{-3}$ and the composition, $60 \% \mathrm{O}, 40 \% \mathrm{C}$. The calculation carried 60,000 zones with a resolution of $100 \mathrm{~cm}$.
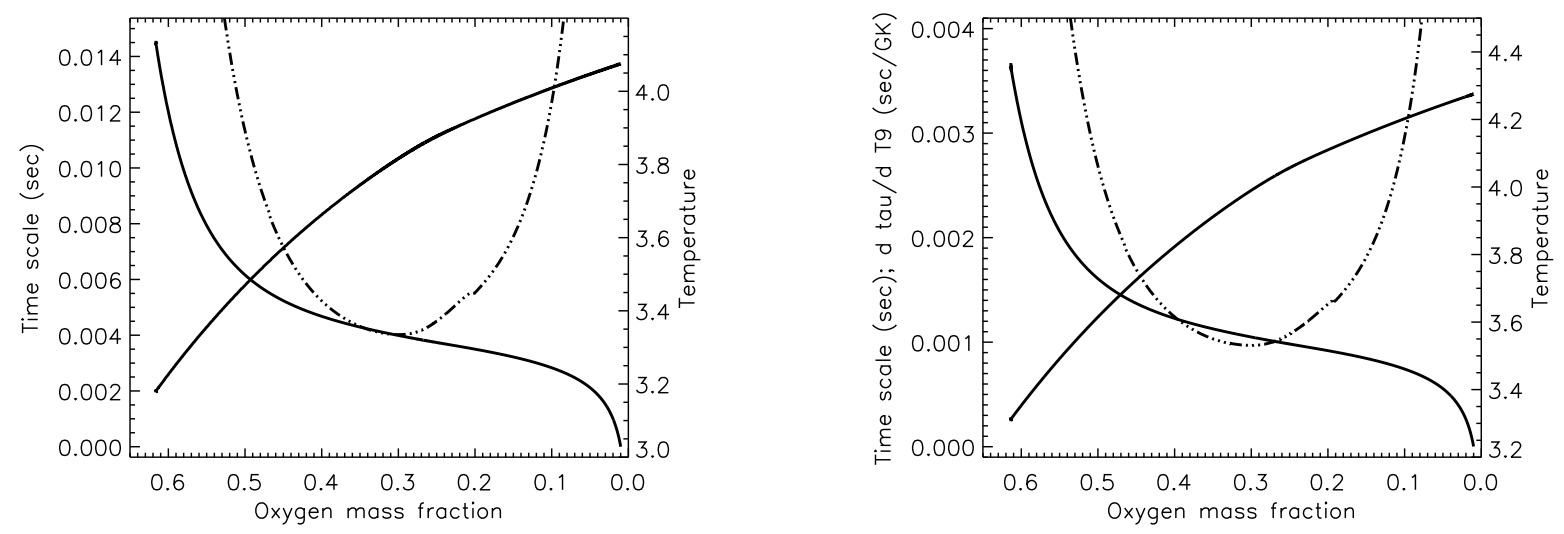

Fig. 4.- Time scale remaining until the remaining oxygen mass fraction is only $1 \%$ plotted as a function of the remaining oxygen mass fraction (solid line). The density of the carbonoxygen fuel is $2.5 \times 10^{7} \mathrm{~g} \mathrm{~cm}^{-3}$ (left frame) or $3.0 \times 10^{7} \mathrm{~g} \mathrm{~cm}^{-3}$ (right frame). Also given is the temperature and the derivative, $d \tau / d T_{9}$, of the time scale with respect to temperature (dashed line). Multiplying this derivative by the spread of temperatures in a region, L, gives approximately the range of times during which the fuel in that region will be exhausted. The initial composition was $60 \%$ oxygen and $40 \%$ carbon by mass and the initial temperature, $6 \times 10^{8} \mathrm{~K}$, but only the evolution after carbon depletion is plotted. 
Still the results found here are encouraging, and the necessary multi-dimensional simulations are feasible.

The authors gratefully acknowledge helpful conversations on the subject of the paper with John Bell. This research has been supported by the DOE SciDAC Program (DEFC02-06ER41438); the National Science Foundation (AST 0909129); and the NASA Theory Program (NNX09AK36G). Work at Sandia was supported by the US Department of Energy, Office of Basic Energy Sciences, Division of Chemical Sciences, Geosciences and Biosciences. Sandia is a multiprogram laboratory operated by Sandia Corporation, a Lockheed Martin Company, for the US Department of Energy under contract DE-AC04-94AL85000. Work at LBNL was supported by the Applied Mathematics Research Program of the U.S. Department of Energy under Contract No. DE-AC02-05CH11231.

\section{REFERENCES}

Aspden, A. J., Bell, J. B., Day, M. S., Woosley, S. E., \& Zingale, M. 2008, ApJ, 689, 1173

Aspden, A. J., Bell, \& Woosley, S. E. 2010, ApJ, 710, 1654

Damköhler, G. 1940, Z. Elektrchem, 46, 601

Hoflich, P., Khokhlov, A. M., \& Wheeler, J. C. 1995, ApJ, 444, 831

Kasen, D., Röpke, F. K., \& Woosley, S. E. 2009, Nature, 460, 869

Kerstein, A. R. 1991, J. Fluid Mech., 231, 361

Kerstein, A. R. 2001, Phys. Rev. E., 64, 066306

Khokhlov, A., Oran, E. S., \& Wheeler, J. C. 1997, ApJ, 478, 678

Landau, L., \& Lifshitz, F. M. 1959, Course in Theoretical Physics, Vol. 6. Fluid Mechanics (Oxford:Pergammon)

Mazzali, P. A., Röpke, F. K., Benetti, S., \& Hillebrandt, W. 2007, Science, 315, 825

Niemeyer, J. C., \& Woosley, S. E. 1997, ApJ, 475, 740

Peters, N. 1986, Proc. Combust. Inst., 21, 1231

Plewa, T., Calder, A. C., \& Lamb, D. Q. 2004, ApJ, 612, L37 
Röpke. F. 2007, ApJ, 668, 1103

Timmes, F. X., Hoffman, R. D., \& Woosley, S. E. 2000, ApJS, 129, 377

Timmes, F. X., \& Swesty, F. D. 2000, ApJS, 126, 501, see also http://cococubed.asu.edu/code_pages/eos.shtml

Woosley, S. E., Kerstein, A. R., Sankaran, V., Aspden, A. J., Röpke, F. K. 2009, ApJ, 704, 255, Paper 1 
Table 1. Oxygen Burning Timescales

\begin{tabular}{ccccccc}
\hline \hline $\mathrm{X}_{12}$ & $\begin{array}{c}\rho \\
\left(10^{7} \mathrm{~g} \mathrm{~cm}^{-3}\right)\end{array}$ & $\begin{array}{c}T_{\mathrm{C}-\text { ash }} \\
\left(10^{9} \mathrm{~K}\right)\end{array}$ & $\begin{array}{c}\rho_{\mathrm{C}-\text { ash }} \\
\left(10^{7} \mathrm{~g} \mathrm{~cm}^{-3}\right)\end{array}$ & $\mathrm{X}_{16}$ & $\begin{array}{c}\tau_{16} \\
(\mathrm{sec})\end{array}$ & $\begin{array}{c}T_{\mathrm{O}-\text { ash }} \\
\left(10^{9} \mathrm{~K}\right)\end{array}$ \\
\hline 0.30 & 2.25 & 2.79 & 1.61 & 0.714 & $2.64(-1)$ & 3.93 \\
0.30 & 2.50 & 2.85 & 1.80 & 0.713 & $1.18(-1)$ & 4.04 \\
0.30 & 2.75 & 2.91 & 2.00 & 0.712 & $5.72(-2)$ & 4.14 \\
0.30 & 3.00 & 2.96 & 2.21 & 0.711 & $2.98(-2)$ & 4.23 \\
0.30 & 3.25 & 3.01 & 2.41 & 0.710 & $1.66(-2)$ & 4.32 \\
0.30 & 3.50 & 3.05 & 2.61 & 0.709 & $9.54(-3)$ & 4.40 \\
0.30 & 3.75 & 3.10 & 2.81 & 0.708 & $5.77(-3)$ & 4.48 \\
0.30 & 4.00 & 3.14 & 3.02 & 0.707 & $3.62(-3)$ & 4.54 \\
0.30 & 4.25 & 3.18 & 3.22 & 0.706 & $2.34(-3)$ & 4.62 \\
& & & & & & \\
0.40 & 1.75 & 2.94 & 1.11 & 0.621 & $2.56(-1)$ & 3.71 \\
0.40 & 2.00 & 3.03 & 1.29 & 0.619 & $8.59(-2)$ & 3.84 \\
0.40 & 2.25 & 3.11 & 1.48 & 0.618 & $3.37(-2)$ & 3.97 \\
0.40 & 2.50 & 3.18 & 1.66 & 0.616 & $1.48(-2)$ & 4.08 \\
0.40 & 2.75 & 3.25 & 1.85 & 0.615 & $7.13(-3)$ & 4.18 \\
0.40 & 3.00 & 3.32 & 2.04 & 0.614 & $3.70(-3)$ & 4.28 \\
0.40 & 3.25 & 3.37 & 2.23 & 0.612 & $2.06(-3)$ & 4.37 \\
& & & & & & \\
0.50 & 1.50 & 3.05 & 0.856 & 0.530 & $3.89(-1)$ & 3.59 \\
0.50 & 1.75 & 3.16 & 1.02 & 0.528 & $1.04(-1)$ & 3.74 \\
0.50 & 2.00 & 3.26 & 1.20 & 0.526 & $3.44(-2)$ & 3.88 \\
0.50 & 2.25 & 3.36 & 1.37 & 0.525 & $1.33(-2)$ & 4.00 \\
0.50 & 2.50 & 3.44 & 1.54 & 0.523 & $5.87(-3)$ & 4.12 \\
0.50 & 2.75 & 3.52 & 1.72 & 0.521 & $2.85(-3)$ & 4.22 \\
0.50 & 3.00 & 3.59 & 1.90 & 0.520 & $1.51(-3)$ & 4.32 \\
\hline
\end{tabular}

